various intervention sectors where work conditions are often difficult. Once juxtaposed to the characteristics attached to disasters (nature, suddenness, duration, intensity, etc), the characteristics of the workers (intervention skills, training received, intrinsic efforts made, etc) and to the characteristics of the organizations (expectations towards their employees, organizational support offered to the employees, extrinsic efforts required, etc), these conditions increase their level of vulnerability by exposing them to environments harsh to manage. This vulnerability experienced by the workers in an emergency period can be reflected through symptoms such as anxious disorders and exhaustion. This poster will present the major findings of recent studies in this field (impact of disaster on the psychological health of workers) while under lighting personal, contextual and organizational factors which either contribute to the presence of psychological health issues for the workers or facilitate their resilience.

Prehosp Disaster Med 2011;26(Suppl. 1):s116-s117

doi:10.1017/S1049023X11003888

(P1-57) Develop New Mechanism of Capacity Building of Disaster Preparedness in China W. Zhang, ${ }^{1}$ B. Huang, ${ }^{2}$ S. Miao, ${ }^{3}$ J. Yao, ${ }^{1}$ B. Zhang, ${ }^{1}$ Q. Li $^{1}$

1. Office for Disease Control and Emergency Response, Beijing, China

2. Jinan, China

3. Tianjin, China

Background: China is one of the countries most affected by natural disasters, it is an important restricting factor for economic and social development. However, Disaster Medicine training is not included in medical education curriculum in China, continual training is separated among public health professionals and clinical personals.

Methods: WHO provides technical and financial support for public health emergency preparedness through intensive training and workshop. We intended to develop a new working mechanism under the support of $\mathrm{WHO}$ and $\mathrm{MOH}$, China for capacity building of disaster preparedness in China with the combination of public health professionals and clinical personals though TOT training.

Results: Through the new mechanism, public health professionals from CDC system and clinical personals from hospitals could benefice mutually fro each side and strengthen the effectiveness for the disaster preparedness.

Conclusion: The new mechanism increases the effectiveness of capacity building for disaster preparedness, TOT training should transit from national level to local level.

Prehosp Disaster Med 2011;26(Suppl. 1):s117

doi:10.1017/S1049023X1100389X

(P1-58) Frailty, Dementia and Disaster: An E-Learning Initiative for Health Care Providers

D. Maltais, ${ }^{1}$ M. Gibson, ${ }^{2}$ L. Hardy, ${ }^{3}$ S. Ruthe, ${ }^{4}$ M. Balazs ${ }^{2}$

1. Human Science, Chicoutimi, Canada

2. London, Canada

3. Senior Services/Adult Protection, Yukon, Canada

4. Community Emergency Preparedness, Victoria, Canada

Frailty, Dementia and Disaster: an e-learning initiative for Health care providers D. Maltais ${ }^{1}$, M. Gibson ${ }^{2}$, L. Hardy ${ }^{3}$, S. Ruthe ${ }^{4}$, ${ }^{1}$ nniversity of Quebec, Chicoutimi, ${ }^{2}$ St. Joseph's
Health Care London, London, ${ }^{3}$ Health and Social Services, Yukon Territory Government, Whitehorse, ${ }^{4}$ Emergency Program, Corporation of the District of Oak Bay, Victoria, Canada.

This poster describes the development, piloting, evaluation, and dissemination of the e-learning tool: "Frailty, Dementia and Disasters: What Health Care Providers Need to Know". The purpose of the e-learning tool is to contribute to international efforts to reduce the disproportionate vulnerability of older adults in emergencies and disasters. Key literature on geriatric emergency preparedness and response issues, including the roles and responsibilities of health care providers, was identified and synthesized. Content was piloted in a facilitated workshop in Ontario. A Canada-wide health provider reference group provided feedback on the transition from a traditional powerpoint presentation to an e-learning format. The evaluation process included facilitated review of the tool by health care providers in two in-person workshops in each of Yukon and Quebec and in an independent review by health care providers in British Columbia (virtual). The learning objectives of the e-learning resource are to help health care providers, administrators and policy makers understand the: - disproportionate vulnerability of older adults who are frail and those who have dementia, in emergencies and disasters; ' components of the emergency management cycle and how they apply to this target population; $\cdot$ best practice resources that can be used to improve emergency preparedness, response, recovery and mitigation; and · role of health care organizations and providers in emergency management for these older adults. The e-Learning tool is hosted with open access on www.dementiaknowledgebroker.ca (DKB), a platform facilitated by the CDRAKE - the knowledge exchange theme of the Canadian Dementia Knowledge TranslationNetwork (CDKTN).

Prehosp Disaster Med 2011;26(Suppl. 1):s117

doi:10.1017/S1049023X11003906

\section{(P1-59) Atom Course in Japan}

T. Nagata, ${ }^{1}$ M. Hashizume, ${ }^{1}$ A. Lefore ${ }^{2}$

1. Department of Emergency and Critical Care Center, Fukuoka, Japan

2. Department of Surgery, Oyama, Japan

Introduction: Trauma care is one of the key components of disaster medicine. However, it is difficult in Japan to gain extensive experience in trauma surgery, especially penetrating trauma. The Advanced Trauma Operative Management (ATOM) course was developed as a model for teaching operative trauma techniques to surgical residents, fellows, and attending surgeons as the number of these cases decreases in the US. In 2008, a new ATOM training site was established at Jichi Medical University in Japan, and as of December, 2010, five courses have been offered.

Methods: The ATOM course consists of lectures and a porcine operative experience. Comprehensive evaluation of ATOM was designed to assess participant learning in the cognitive, affective, and psychomotor domains. Data on the first 36 participants was retrospectively collected and analyzed.

Results: Participants included: 20 expert trauma surgeons, and 16 general surgeons. All groups showed improvement in knowledge (pre-test score: $61.9 \pm 16.4$ (mean \pm standard deviation), post-test score: $70.6 \pm 16.5$, $p$-value $<0.001)$ with results in the expert and fellow groups reaching statistical significance. 
Self-confidence also improved (pre-evaluation score: $65.4 \pm$ 17.6 , post-evaluation score: $82.0 \pm 9.4, p$-value $<0.001)$, with all groups reaching statistical significance.

Discussion: This course creates real operative situations in a standardized fashion that improves knowledge and operative confidence for trauma operations, which may be of great benefit in disaster medicine training.

Prehosp Disaster Med 2011;26(Suppl. 1):s117-s118

doi:10.1017/S1049023X11003918

\section{(P1-60) Clinico-Epidemiological Pattern and Management Options in Patients with Ocular Injuries in the Emergency Room of a Rural Hospital \\ S. Baisakbiya, ${ }^{1}$ M. Bhatt, ${ }^{2}$ A. Agrawal ${ }^{3}$ \\ 1. Ophthalmology, Mullana (Ambala), India \\ 2. Anesthesiology, Ambala, India \\ 3. Neurosurgery, Ambala, India}

Aims and Objectives: Successful patient outcomes in the setting of ocular emergencies depend on correct recognition and assessment as well as appropriate initial management. The purpose of this study is to describe the clinico-epidemiological findings and management options in patients with ocular injuries in the emergency room of a rural hospital.

Material and Methods: In this retrospective review the records of patients who were treated for ocular trauma from June 2010 to December 2010 in the emergency room of MM Institute of Medical Sciences and Research, Mullana (Ambala) were reviewed. The following data for all patients were recorded: age, sex, date and time of injury, involved eye, circumstance and mechanism of injury, initial visual acuity, details of appropriate investigation, immediate management and outcome.

Results: A total 46 patients were included in the study. Young adult male patients were more commonly involved. Most of the patients presented within 12 hours of injury. Left eye was involved in 14 patients; right in 12 and 10 patients had injuries to both eyes. Most common mode of injury was mechanical. The patients who sustained bilateral ocular injuries were due to chemical burns ( 7 cases) and electrocution (3 cases). Most of the patients were managed conservatively. The surgical treatment offered were repair of corneal tears, removal of foreign bodies. The visual outcome was excellent in most of the patients.

Conclusions: Ocular injuries can have wide range of etiological factors and presentation and assessment of ocular emergencies can be made difficult by a lack of sophisticated facilities. However, a concise patient history, general observation and basic ocular tests can lead to a firm diagnosis and thereby appropriate management.

Prehosp Disaster Med 2011;26(Suppl. 1):s118

doi:10.1017/S1049023X1100392X

(P1-61) Nursing Education for Disaster Management J. Boone, ${ }^{1}$ D. Moore 2

1. Director of Global Studies, Ontario, United States of America

2. Nursing, Coast Mesa, United States of America

In Southern California in 2010, there were 76 declared disasters of which the majority were in Southern California, for this reason and at the request of our clinical partners, when
West Coast University (WCU) started its nursing program in 2008, it recognized the need to have a course in disaster management. With nursing being the largest of the health professions, the American Association of Colleges of Nursing (AACN) recognized the need to incorporate disaster nursing in its new Baccalaureate Nursing standards. The Disaster Management course was developed in concert with other parts of the curriculum such as Leadership, Physical Assessment, Critical Care courses to help students focus their assessment and intervention skills to prepare them to be future responders. The disaster management course is delivered in both didactic and hands on format including online Federal Emergency Management Agency (FEMA) modules, disaster drills, guest lecturers and field trips. Students are also introduced to many volunteer opportunities and are trained to be disaster ready once they are needed. Students are excited and ready to volunteer at various organizations before graduation such as the American Red Cross, the Medical Volunteer Program and the Disaster Medical Assistance Teams. Since WCU has new campuses, the students have been instrumental in implementing a new disaster plan for our facilities, including developing flyers, videos and conducting disaster drills. We have received feedback from our clinical partners that our students are better prepared than their current emergency staff in regards to disaster management and to that end we plan to work with our clinical partners to translate our class into an online course so their staff can be trained on disaster management.

Prehosp Disaster Med 2011;26(Suppl. 1):s118 doi:10.1017/S1049023X11003931

(P1-62) The Risk Factors for Difficult or Failed Airway: A Prosepctive Cohor Study

C. Hsu

Department of Emergency Medicine, Taipei, Taiwan

The risk factors for difficult airway or failed airway: a prospective cohort study Airway management is always the first priority and time-treasures in critical ill-patients. Improper management of difficult airway or resultant fail airway would bring poor prognosis to patients. We investigated the risk factors of difficult or fail airway from the multiple dimension of factors including patients, healthcare and airway devices. We enrolled 252 intubated patients, including 37 trauma patients, 55 patients $(22 \%)$ with difficult airway, and 22 patients $(8.7 \%)$ with fail airway. In analysis of risk factors of difficult airway, factors including obesity, short neck or thickness of soft tissue, facial deformities and oral-nasal bleeding have positive association with fail airway, but the seniority of healthcare providers had no effect. However, experienced healthcare providers have more success rate after the occurrence of fail airway. The most complications of fail airway include airway trauma and hypoxia. As compared with non-trauma patients, trauma patients have more episodes of fail airway, difficult airway, and use of RSI, rescue airway for fail airway, airway trauma and vomiting. Therefore, it is necessary to establish an easy and safe standard guideline in daily practice of difficult and urgent airway management for healthcare providers.

Prehosp Disaster Med 2011;26(Suppl. 1):s118

doi:10.1017/S1049023X11003943 